\title{
NOTE SUR L'ACCLIMATATION \\ DU GAMBUSIA HOLBROOKI \\ DANS LES ÉTANGS DES LANDES
}

\author{
par P. CHIMITS \\ Inspecteur des Eaux et Forets
}

En r94ı, étant alors au Laboratoire du Service de la pèche au Musée de la Mer, à Biarritz, mon attention fut attirée sur un pêcheur qui employait comme vif dans les étangs de Biarritz, des petits poissons inconnus', longs de quelques centimètres seulement.

Le pêcheur déclara en trouver en quantité dans les marais de Geloux, à 15 kilomètres à l'ouest de Mont-de-Marsan.

Je déterminai ces petits poissons comme étant Gambusia Holbrooki (Ginaro) ; petit Cyprinodonte originaire du sud des Etats-Unis. Comment étaient-ils venus d'Amérique à Mont-de-Marsan ? (Fig. 13).

Un article de M. le Professeur E. Bnumpt, de la Faculté de Médecine de Paris, m'apprit que le Gambusia Holbrooki avait été importé en I92 I des Etats-Unis en Espagne, dans la régjon de Barcelone, pour la lutte antipaludique. Il y réussit fort bien, s'y développa en abondance et il en fut exporté, dans le mème but, de nombreux exemplaires en Italie en 1922.

C'est d'Italie qu'en rgá il fut introduit en Corse. M. le Professeur BruMpt, en 1926, constata les merveilleux résultats donnés par le Gambusia dans la destruction des larves de moustiques et le répandit dans toute la Corse.

J'écrivis alors à M. Ic Professeur Brumpt, qui me répondit en effet avoir, depuis I927, introduit le Cambusia avec succès dans la région méditerranéenne, et, en 1931 , en avoir déversé quelques exemplaires dans les marais proches de Mont-de-Marsan et à l'étang de Biscarosse, sans avoir pu revenir sur les lieux constater les résultats de son opération.

J'en élevai en r942 quelques exemplaires au Musée de la Mer et constatai que ces petits poissons vivipares se reproduisaient fort bien en aquarium, en eau douce comme en eau salée.

En $1943, j$ jen déposais deux couples dans chacun des étangs de la Prade, de Moliets et de Léon, situés à environ 5o kilomètres de Biarritz, sur la côte des Landes. Ils proliférèrent abondamment, et dans l'été $1945 \mathrm{~d}$ 'innombrables Gambusies étaient visibles sur les bords de ces étangs.

Il ne mappartient pas, surtout après les travaux de $M$. le Profosscur Brựpt, d’insister sur la grande utilité des Gámbusies dans la lutte contre 
les larves de moustiques - je rends compte simplement de quelques observations que $\mathrm{j}$ 'ai pu faire à ce sujet.

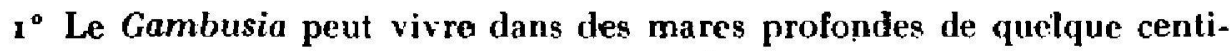
mètres.

Dans les marais de Geloux j'en ai compté des centaines d'individus, très serrés dans une mare presque desséchée de quelques mètres carrés.

L'eau stait très acide ( $p H=5,5$-degré hydrotimétrique $\left.r^{\circ} \mathbf{j}\right)$ et très chaule $\left(32^{\circ}\right.$ en surface). Les Gambusies n'avient pas l'air d'en souffrir et, d'ailleurs, l'espèce s'y perpétuait depuis to ans.

J'ai pu constater, l'été a 947 , la présen-

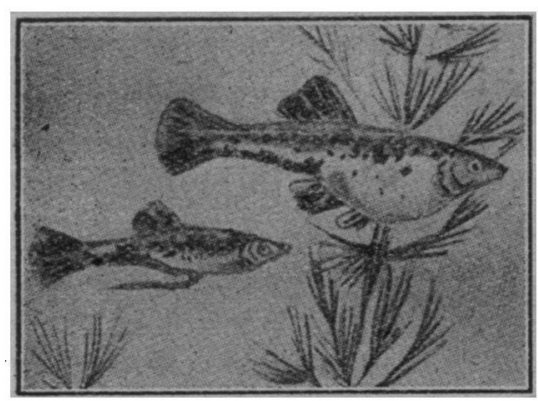

Fíg. 13. - Couple de Cambusa Molbrooki. re de Gambusia dans des mares où la température atteignait $36^{\circ} \mathrm{C}$.

$2^{\circ}$ Le Gambusia se transporte très facilement et sans soins spéciaux - c’est ainsi que cet été 1947 j'ai porté trois sujets mis dans une boîte de conserve contenant $1 / 4$ de litre d'eau ct placé dans une valise, de Dax à Paris - ils nont souffert en rien de ce voyage de près de 12 heures.

$3^{\circ}$ Un Gambusia de a à 3 centimètres de long dévore une moyenne de ro à ij

larves de moustiques par jour. Il est facile de le constater en comptant les larves 'mises en aquarium avec un Gambusia. D'ailleurs, il ne s'attaque. qu'aux larves vivantes et en mouvement.

Ses yeux placés sur la partie supérieure de la tête, lui permetlant de voir au^dessus de lui et de déceler les mouvements des larves de moustiques venant respirer à la surface de l'eau; repu, il ne s'attaque plus aux larves.

Donc pendant unc période estivale de 120 jours, un Gambusia peut dévorer une moyenne de 1.500 larves. Si l'on comple qu'une femelle pond plusieurs générations par an, on comprend qu'une mare puisse être rapidement nettoyée de ses larves de moustiques.

$4^{\circ}$ Le Gambusia devient cannibale et dévore ses petits âgés de quelques jours, si on le laisse jeûner 2 ou 3 jours.

$5^{\circ}$ Pendant l'été I94r, j'ai constatẹ́ qu'il était très difficile de trouver une larve de moustique sur les bords des étangs de la Prade et de Moliets. En revanche, dans les mares pluviales voisines, non permanentes, où le Gambusia n avait pas été introduit, les larvos de moustiques pullulaient, à raison de 3 à 400 par mètre carré.

De même, à l'étang de Moliets, une barque au fond rempli d'eau contenait de nombreuses larves de moustiques ( 5 à 6 par décimètre carré) alors que les eaux de l'étang $n$ 'en contenait que très peu. l.'introduction, dans 
la barque de 6 Gambusies, a suffi pour purger presque complètement le bateau de ses larves d'Anophèles en ro jours.

$6^{\circ}$ L'hiver, le Gambusia semble disparaittre ; peut-ètre périt-il en grande quantité : Au printemps, aux pretniers rayons de soleil, quelques gros individus réapparaissent ; la requoduclion semble devoir commencer quand la température de l'eau atteint 16 à $18^{\circ}$, c'est-à-dire, dans les étangs landais, vers la fin du mois d'Avril.

Je signale qu'en Juillet 1947 , jai livré à l'inspecteur des Eaux et Forèts à Dax, des Gambusies pour les déverser dans les mares voisines de Dax, pour y détruire les mousliques si désagréables pendant les nuits d'été.

II n'est pas plus difficile en effet de mettre 3 ou 4 couples de Gambusies chaque année dans une mare que d'y déverser du pétrole. Il semble que deux ou trois hommes, munis cliacun d'une épuisette et d'un bidon, peuvent en trós jours ensemencer convenablement tous les étangs, mares el flaques mêmes non permanentes entourant une vilte.

Mais je voudrai surtout insister sur l'utilité du Gambissia dans l'amé. nagement des lacs à Perches. Il est de fait que, dans la plupari des lacs à Perches (perca fluviatilis) et notamment les lacs des Landes, la Perche a tendance au nanisme.

On a parlé à ce sujet de la dégtinérescence de la Perche. Il est très probable que le nanisme est dù simplement à la prolifération excessive de la Perche dont les jeunes sujets sont arrêtés clans leur développement, par le manque de nourriture appropriée.

Or, dans nos lacs des Landes, lo jeune alevin peut se nourrir au tlébut de son existence de larves, dinsectes et d'alevins, mais il arrive un moment où les alevins de Gardons et surtout de Perches-soleil deviennent trop gros pour la touche de nos perchettes qui restent inféfiniment à une taille d'une donzaine de contimètres et d'un poids de vingrt à cinfuante grammes. C'est, en somme, le phénomène qui se produit dans un étang d'éleinage surchargé en aleving de Carpes.

Lne solution pour arriver à "refaire " des Perches convenables d'an moins 150 grammes, consiste à permettre la pêche des petites Perches, en dessous de la taille réglementaire, pour en diminuer le nombre et l'adapter à la quantité de nourriture offerte, par le lac.

I ne: autre solution consiste à augmenter la nourriture rationnelle des perchettes. Et une nourriture excellente est précisément fołrnie par le Cambusia.

Dans les étangs de Léon, Molieks et la Prade, le Gambusia forme une auréole sur lout le lord utilisant pour nourriture les larves sur une profondeur de quelques centimètres inaccessible aux autres poissons. Les Gambusies utilisent donc au meux la nourriture naturelle offerte sur ces minces couches d'eau qui était pratiquement inemployées avant leur introduction. Ils ne nuisent donc pas à la nourriture des autres poissons. 
Les Gambusies sont une proie particulèrement facile et abondante pour les perchetles, tant par leur petite taille que par le fait qu ils se promènent toujours en surface et ne voient pas le vorace venant en dessous d'eux.

De fait, j'ai fréquemment trouvé des Gambusies dans le ventre des Perches.

D'autre part, j'ai pu constater, notamment à l'étang de la Prade en 1946 et 1947 , que la taille des Perches capturées à la ligne avait sensiblement augmenté. Alors que la Perche de plus de 100 grammes étail très rare jusqu'en I943, j'ai capturé en 1946 et 1947 d'assez nombreuses Pèrches de 150,200 et 250 grammes que l'on ne capturait plus depuis bien longtemps.

Sans doute ces observation auraient-elles besoin d'être suivies de beaucoup d'autres, mais je crois utile de signaler, dès à présent, que l'introduction du Gambusia dans les lacs chauds augmente en une grande proportion et de façon, si je puis dire "gratuite ", la nourriture offerte aux voraces et notamment à la Perche, et que cette introduction est susceptible de présenter à l'inquiétant problème piscicole posé par la 'pullulation des Perches naines dans certains lacs, une solution qui peut être combinée avec l'abaissement de la taille réglementaire de cette espèce, mais qui n'en est pas moins élćgante.

En résumé, les trois points suivants peuvent être considérés comme acquis en ce qui concerne les étangs du Sud-Ouest de la France :

$I^{\circ}$ Le Cambusia Holbrooki y est délinitivement acclimaté et fait partie de la faune piscicole normale.

$2^{\circ}$ ll constitue, jusqu'à présent au moins, une composante intéressante de la Sitesse itchtyenne, utilise la zonc marginale des étangs et scrt principałement de nourriture aux jeunes perchettes.

$3^{\circ}$ Il peut etre facilement utilisé autour des villes du Sud-Ouest pour combattre, non le paludisme qui n'y existe pas, mais les moustiques qui présentent un inconvénient sérieux pour le sommeil des habitants. Je signale notamment les alentours marécageux des villes de Bordeaux, Bayonne et Biarritz.

Enfin, et à ce jour, il n'a pas été constaté d'inconvénient piscicole à ła présence du Gambusia, bien que nous ayons pu observer en aquarium, que ce roisson privé de nourriture, devorait ses petits àgés de quelques jours.

\section{OUVRAGES CONSULTÉS}

1928. - Professeur Bnumpt (E.).-- C. R. Sciences, T. 189, p. 909.

1928. - Professeur Selka. - Bolletimo di pesca di piscicollura et di idrobiogia, II I, IV.

1929. - Hugh M. Smitr. - Journal of the Siam Society Natural History, VIII, I.

1930. - Professeur Brumpt (E.). - Siècle Médical, no de Mars, p. 3.

1931. -.. Suzanne houle. …B. F. P., no 33, p. 157.

1934. - Manuel DagnY. - B. F. P., no 79 , p. 178. 